\title{
Invasion-inhibiting Effects of Gaseous Components in Cigarette Smoke on Mouse Rectal Carcinoma Colon-26 Cells
}

\author{
MAYUKO HATAI, NORIKO YOSHIKAWA, ERIKO KINOSHITA, SHIZUYO HORIYAMA, \\ SATOMI KAGOTA, KAZUMASA SHINOZUKA and KAZUKI NAKAMURA \\ Department of Pharmacology, School of Pharmacy and Pharmaceutical Sciences, \\ Mukogawa Women's University, Nishinomiya, Japan
}

\begin{abstract}
We investigated the anti-metastatic action of nicotine- and tar-removed cigarette smoke extract (CSE) on highly metastatic mouse Colon-26 cells using syngeneic $B A L B / c$ mice. Colon-26 cells were injected into the spleen of mice, cells were grown in the spleen as the primary lesion, and some metastasized from the spleen to liver and established a metastatic lesion. CSE (10,30, and 100\%) was intraperitoneally administered daily to the mice for 14 days after tumor inoculation. As a result, the relative spleen weights of CSE-administered mice did not differ significantly from those of the control mice. However, the relative liver weights of CSE 30\%-administered mice significantly decreased compared to control mice. In order to identify the active component in CSE, we examined the action of methyl vinyl ketone (MVK) on the invasiveness of Colon-26 cells. MVK significantly reduced the invasiveness of cells. MVK may be a candidate active component of CSE.
\end{abstract}

Cigarette smoking is one of the worst habits for human health. Although cigarette smoke on the whole, including nicotine and tar, is very harmful, it is possible that some of the components in cigarette smoke may be beneficial (1). In fact, a total of 3,996 constituents have been isolated from the mainstream smoke of cigarettes (2). Cigarette smoke contains both gaseous and particulate components, and the latter components (tar) and nicotine have been investigated in detail by numerous researchers (3-7). Therefore, we

This article is freely accessible online.

Correspondence to: Mayuko Hatai, Department of Pharmacology, School of Pharmacy and Pharmaceutical Sciences, Mukogawa Women's University, 11-68, Koshien Kyuban-cho, Nishinomiya, Hyogo 663-8179, Japan. Tel/Fax: +81 798459945, e-mail: hatai@mukogawa-u.ac.jp

Key Words: Nicotine- and tar-removed cigarette smoke extract, CSE, mouse rectal carcinoma Colon-26 cells, invasion, methyl vinyl ketone, MVK. focused on gaseous components instead of particulate components, and used nicotine- and tar-removed cigarette smoke extract (CSE) in our experiments.

In our previous studies, 3-h pretreatment of highly metastatic mouse melanoma B16-BL6 cells with CSE dosedependently reduced the number of lung metastatic nodules in syngeneic $\mathrm{C} 57 \mathrm{BL} / 6 \mathrm{NCr}$ mice 14 days after tumor injection (8). Furthermore, we demonstrated that methyl vinyl ketone (MVK), a toxic component of CSE, reduced the amount of intracellular glutathione (GSH), the most abundant cellular thiol antioxidant, and showed cytotoxicity to B16-BL6 cells (9).

In this study, we examined the anti-metastatic action of CSE on highly metastatic mouse rectal carcinoma cells (Colon-26 cells) using syngeneic BALB/c mice as an experimental model of metastasis from the spleen to the liver. Moreover, we aimed to identify the active components in CSE by invasion and migration assays in vitro.

\section{Materials and Methods}

Materials. Winston XS Caster FR One Box cigarettes containing 1 $\mathrm{mg}$ of tar and $0.1 \mathrm{mg}$ of nicotine per cigarette were purchased from Japan Tobacco, Inc. (Tokyo, Japan). Cambridge filters were used to remove almost all particles and nicotine from cigarette smoke, and they were obtained from Heinr. Borgwaldt GmbH (Hamburg, Germany). MVK was purchased from Tokyo Chemical Industry Co., Ltd. (Tokyo, Japan). Fetal bovine serum (FBS) and RPMI 1640 medium were from Invitrogen Corp. (Carlsbad, CA, USA). EDTA trypsin solution (EDTA: $2.2 \mathrm{mM}$, trypsin: 0.25\%) was from Mediatech, Inc. (Manassas, VA, USA). Dulbecco's phosphatebuffered saline without calcium and magnesium [DPBS (-)] was from Nissui Pharmaceutical Co., Ltd. (Tokyo, Japan). Matrigel (Corning ${ }^{\circledR}$ Matrigel ${ }^{\circledR}$ Basement Membrane Matrix Growth Factor Reduced, \#356230) and transwells (FALCON ${ }^{\circledR}$ cell culture inserts, \#353097) were purchased from Corning (Corning, NY, USA). Bovine serum albumin (BSA) and fibronectin were purchased from Sigma Chemical Co. (St. Louis, MO, USA).

Animals. Specific pathogen-free female BALB/cCrSlc mice (7 weeks old) were purchased from Japan SLC, Inc. (Shizuoka, Japan) and used in experiments after 1-week acclimation. Mice were 
maintained in an air-conditioned room $\left(23 \pm 2^{\circ} \mathrm{C}\right.$ and $60 \pm 10 \%$ humidity) under an artificial 12-hour light/dark cycle (7:00 a.m.-7:00 p.m.). Food and water were given ad libitum during the experimental period. All procedures followed the Guidelines for the Care and Use of Laboratory Animals at Mukogawa Women's University (approval number: P-11-2014-03-A).

Cells. A mouse cell line derived from rectal cancer (Colon-26 cells) was obtained from RIKEN BioResource Center (Ibaragi, Japan). Colon-26 cells were grown in RPMI 1640 medium containing 10\% heat-inactivated FBS.

Preparation of CSE. Cigarettes were continuously combusted by reduced pressure generated using an aspiration pump (Nippon Rikagaku Kikai Co., Ltd., Tokyo, Japan), with the flow rate set at 1.0 $1 / \mathrm{min}$. The main stream of the cigarette smoke was passed through a Cambridge filter to remove the tar phase and nicotine, and subsequently, bubbled into $15 \mathrm{ml}$ of DPBS (-). The combustion of the cigarette was repeated, unless otherwise specified, until the dry weight of the tar phase trapped on the Cambridge filter reached $150 \mathrm{mg}$ (10). This solution was regarded as $100 \%$ CSE. The CSE was immediately filtered through a $0.22-\mu \mathrm{m}$ filter and stored at $-80^{\circ} \mathrm{C}$, and diluted to the required concentration with DPBS (-) when necessary. The final concentrations of these solutions are expressed as a percentage of the initial content

Assay of experimental hepatic metastasis of Colon-26 cells in mice. Sub-confluent Colon-26 cells were harvested with EDTA trypsin solution and suspended at appropriate densities in DPBS (-). The left lower back of mice was minimally incised and the spleen was exposed under anesthesia with urethane. Cells $\left(1 \times 10^{5 / 50} \mu \mathrm{l}\right)$ were injected into the spleen of syngeneic BALB/cCrSlc mice.

CSE $(0,10,30$, and $100 \%, 16 \mathrm{ml} / \mathrm{kg}$ body weight) was daily administered intraperitoneally to the mice for 14 days after tumor cell inoculation. The date of death of mice in each group was recorded. The spleen and liver were excised and weighed.

Proliferation assay. Cells were seeded at $1 \times 10^{5}$ cells $/ 2 \mathrm{ml}$ in each well of a 12-well culture plate. Cells were treated with different concentrations of $\operatorname{CSE}(0.1,0.3$ and $1 \%)$ and $\operatorname{MVK}(0.3,1,3,10$ and $30 \mu \mathrm{M}$ ), and incubated for 24,48 , and 72 hours in a $\mathrm{CO}_{2}$ incubator at $37^{\circ} \mathrm{C}$. Viable cells were enumerated with a Coulter counter.

Invasion assay. Transwells (6.4-mm diameter) were used with tracked-etched polyethylene terephthalate (PET) membrane filters ( $8-\mu \mathrm{m}$ pore size) coated with $25 \mu \mathrm{g} /$ filter of matrigel basement membrane matrix extracted from Engelbreth-Holm-Swarm mouse sarcoma. Sub-confluent cells were harvested with EDTA trypsin solution and resuspended at appropriate density in DMEM containing $0.1 \%$ BSA. Five-hundred-microliter samples of $5 \times 10^{5}$ cells were placed in the upper-chamber compartments. The lower chambers contained $750 \mu \mathrm{l}$ of DMEM added to $20 \mu \mathrm{g} / \mathrm{ml}$ of fibronectin (Sigma Chemical Co.) as a chemoattractant. CSE (0.03 and $0.1 \%)$ and $\operatorname{MVK}(0.1,0.3$, and $1 \mu \mathrm{M})$ were added to the upper and lower compartments. These concentrations of CSE and MVK did not have cytotoxic effects on Colon-26 cells. After 72 hours of incubation in a tissue culture incubator, non-invading cells on the upper side of the filter were completely removed by wiping with a cotton swab. Cells that had penetrated the matrix protein and adhered to the lower surface of the filter were counted microscopically after fixing and stained with a Diff-Quik kit (Sysmex, Hyogo, Japan).

Migration assay. The migration assay was performed in the same way as the invasion assay, without matrigel coating. Cells $\left(5 \times 10^{4}\right.$ cells $/ 500 \mu \mathrm{l}$ ) were added to the upper-chamber compartments. After 24 hours, cells which had migrated to the lower surface of the filter were stained and counted.

Statistical analyses. The data are expressed as the mean $\pm \mathrm{SE}(\mathrm{n}=3-$ 15). Statistical analyses were performed with the $t$-test and Dunnett's test using PRISM Version 4 (GraphPad Software, Inc., San Diego, CA, USA). Kaplan-Meier survival analysis was used to calculate survival curves, followed by the log-rank test to determine significance of differences in survival using PRISM Version 4. Differences were considered significant at $p<0.05$.

\section{Results}

Effects of CSE on the experimental hepatic metastasis of Colon26 cells in mice. Figure 1A shows the effect of CSE on survival in an experimental model of hepatic metastatic rectal cancer using Colon-26 cells. The average survival of control mice was $24.2 \pm 2.0$ days. The average survival of mice administered 10, 30 , and $100 \%$ CSE was 24.8 $\pm 2.2,30.0 \pm 3.0$, and 25.2 \pm 1.9 days, respectively. The survival time of CSE-administered mice demonstrated no significant difference from that of the control mice. Mice administered 30\% CSE showed a tendency to have a prolonged survival time. The relative spleen and liver weights of Colon-26 cell-injected control mice were significantly higher than those of DPBS (-) non-cell-injected normal mice. The relative spleen weights of mice administered CSE demonstrated no significant difference compared with the control mice (Figure 1B). However, the relative liver weights of mice administered $30 \%$ CSE were significantly lower compared with the control mice (Figure 1C).

Effects of CSE on the proliferation, invasion, and migration of Colon-26 cells in vitro. CSE 0.3 and $1 \%$ significantly inhibited cell proliferation (Figure 2A). CSE 0.03 and $0.1 \%$ significantly reduced tumor cell invasion in a concentrationdependent manner, while CSE did not show any significant effect on cell migration (Figure $2 \mathrm{~B}$ and $\mathrm{C}$ ).

Effects of MVK on the proliferation, invasion, and migration of Colon-26 cells in vitro. MVK $(3,10$, and $30 \mu \mathrm{M})$ reduced cell proliferation in a concentration-dependent manner (Figure 3A). Furthermore, $\operatorname{MVK}(0.1,0.3$, and $1 \mu \mathrm{M})$ significantly inhibited the invasion of cells (Figure 3B) but had no effect on cell migration (Figure 3C).

\section{Discussion}

Nicotine is the most well-known component of cigarette smoke, and cigarette smokers continue to smoke habitually 
A

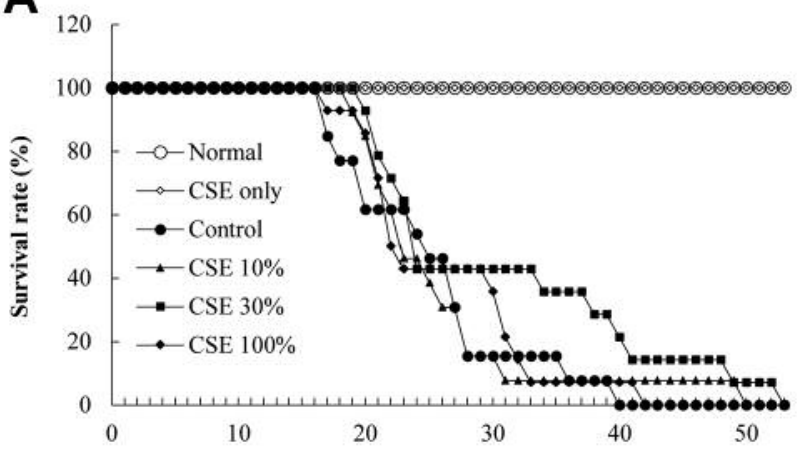

Survival time (days)

B

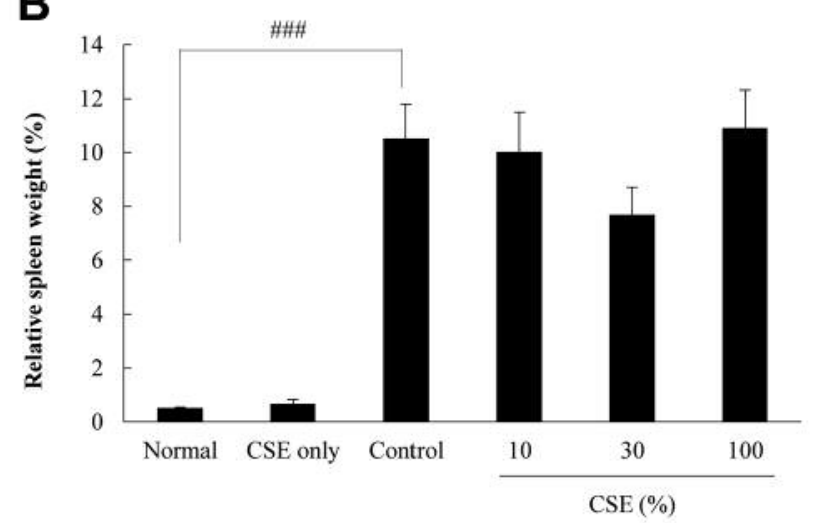

C

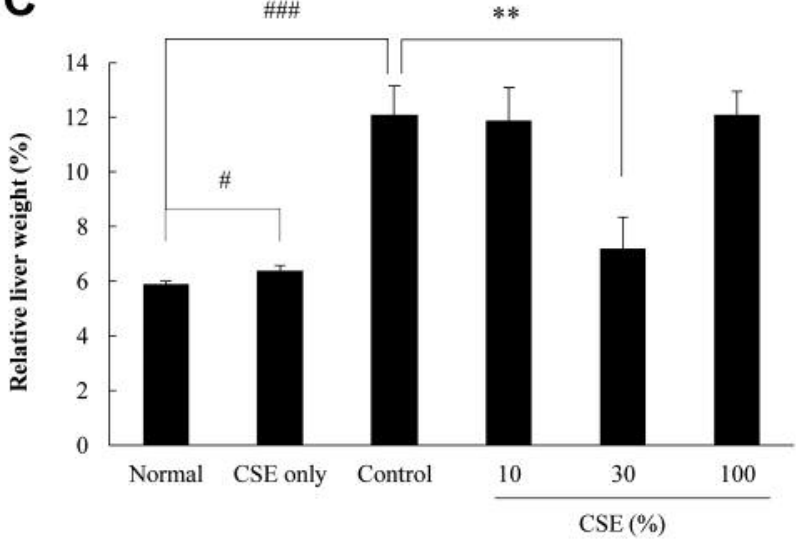

Figure 1. Effects of cigarette smoke extract (CSE) on survival time (A), relative spleen weight $(B)$, and relative liver weight $(C)$ in an experimental mouse model of hepatic metastatic rectal cancer using Colon-26 cells. Non-tumor bearing mice: Normal: administered Dulbecco's phosphate-buffered saline without calcium and magnesium [DPBS (-)], $n=13$; CSE only: administered 100\% CSE, n=7; Tumorbearing mice: Control: administered DPBS (-), n=13; CSE 10\%: administered 10\% CSE, n=13; CSE 30\%: administered 30\% CSE, $n=14$; CSE 100\%: administered 100\% CSE, $n=14$. Data are the

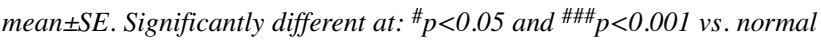
using t-test; ${ }^{* *} p<0.01$ vs. control using Dunnett's test.

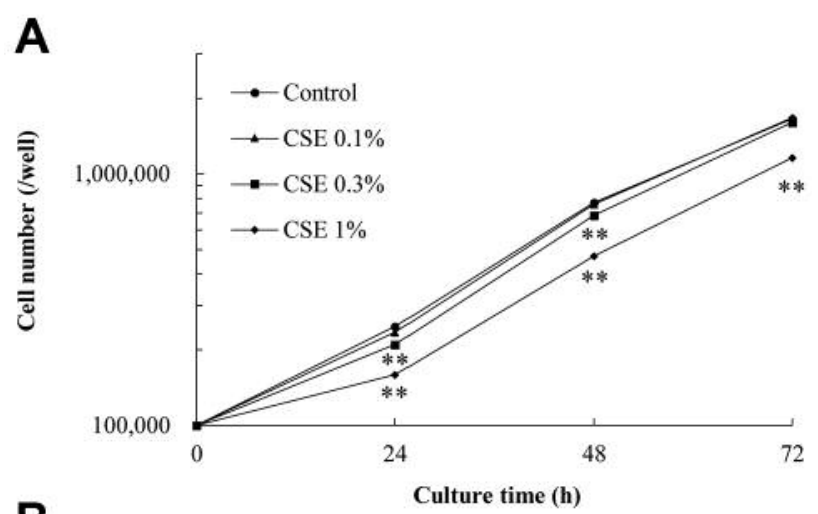

B
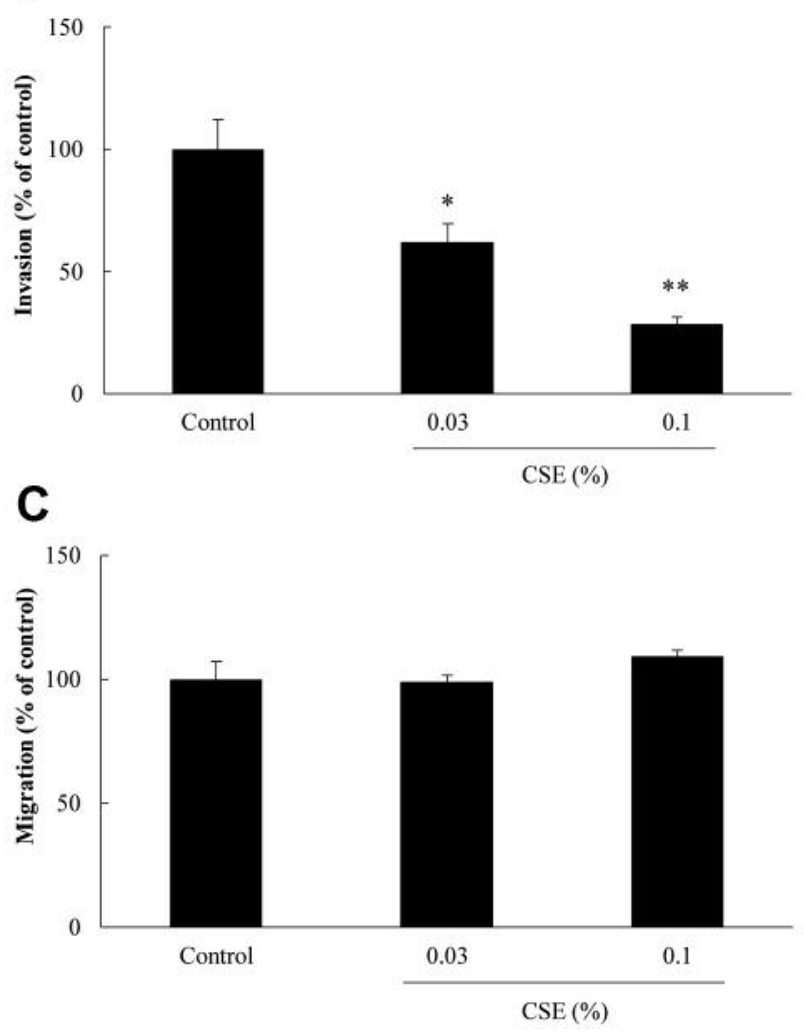

Figure 2. Effects of cigarette smoke extract (CSE) on the proliferation $(A)$, invasion (B), and migration $(C)$ of Colon-26 cells. Data are the mean \pm SE $(n=3)$. Significantly different at: $* p<0.05, * * p<0.01 \mathrm{vs}$. control using the Dunnett's test.

since nicotine creates a physical and psychological dependence (3). Tar is also a well-known component of cigarette smoke, and contains harmful mutagens and carcinogens (5). In our previous study, we focused on components in the gas phase of cigarette smoke, excluding nicotine and tar in the particulate phase, and investigated the pharmacological effect of CSE. We found CSE significantly reduced lung metastatic nodules after the intravenous injection 
A

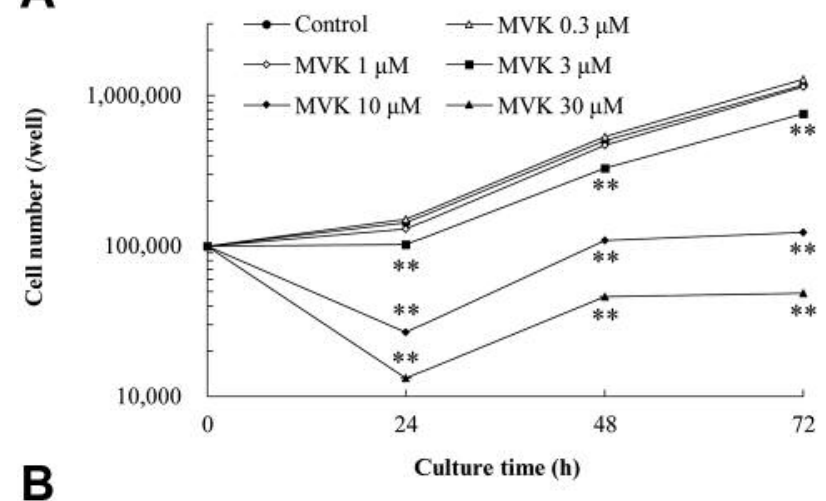

B

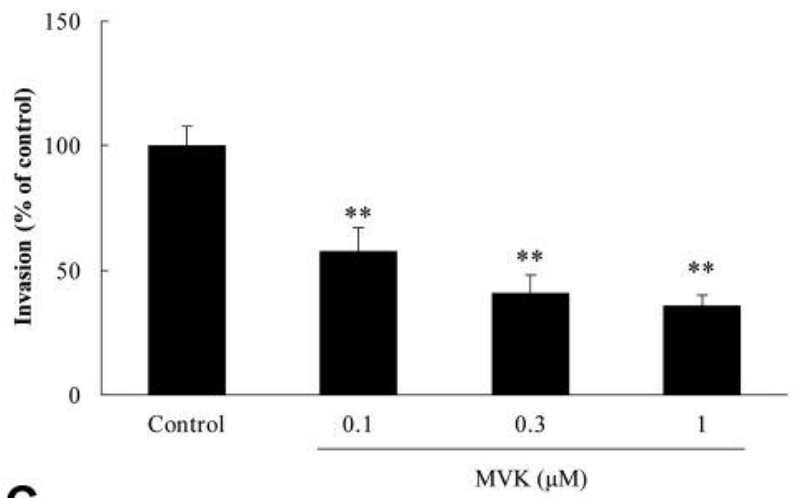

C

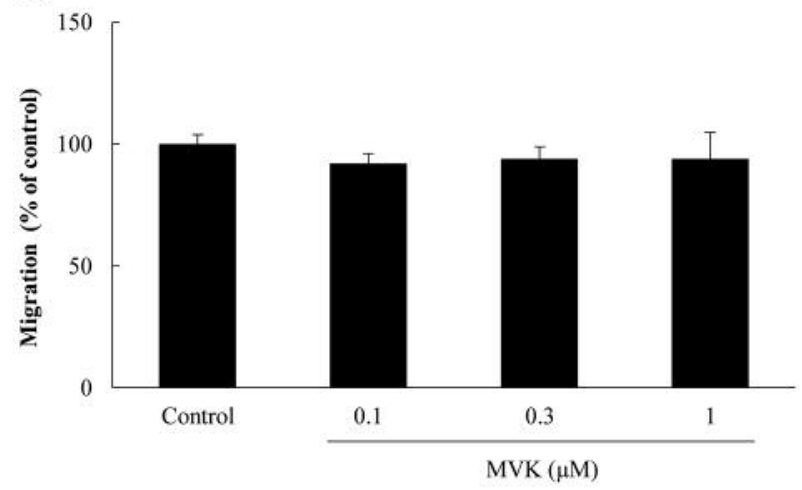

Figure 3. Effects of methyl vinyl ketone (MVK) on the proliferation (A), invasion (B), and migration $(C)$ of Colon-26 cells. Data are the mean $\pm S E$ (proliferation: $n=3$, invasion: $n=8-9$, migration: $n=9-15$ ). Significantly different at: $* * p<0.01 v s$. control using Dunnett's test.

of highly metastatic mouse B16-BL6 melanoma cells pretreated with CSE for $3 \mathrm{~h}$ (8). Actually, CSE was effective and reduced the metastatic potential of B16-BL6 cells even by pretreatment with cells in the flask before injection into the mice. In addition, we used the experimental lung metastatic model as the first step, which lacks the initial metastatic process including departure from the primary lesion, invasion of the extracellular matrix, and entry into veins.
In this study, we investigated the effect of intraperitoneally administered CSE on highly metastatic Colon- 26 cells using a model of metastasis from the spleen to liver. Since human colon tumors show a predilection for the liver as a metastatic organ through the portal vein, we used Colon-26 cells in this mouse model of liver metastasis. An increase in the relative liver weight reflects the establishment of a metastatic lesion comprising Colon-26 cells from the spleen. Although 30\% CSE did not prolong the survival of mice significantly, it had a tendency to prolong the survival time of mice, and significantly reduced the relative liver weight. In fact, $10 \%$ CSE was not effective while 30\% CSE had an anti-metastatic action. This was dose-dependent and reasonable, while $100 \%$ CSE was not effective against tumor metastasis. According to Figure 1C, only mice administered $100 \%$ CSE showed a significant increase in the relative liver weight, and we consider that $100 \%$ CSE had an adverse effect on the liver in mice. Bourgeois et al. demonstrated that the cellular response to CSE exposure is dependent on not only the nominal concentration of CSE, but also on specific experimental variables, including the total cell number, and volume of CSE solution used. They suggested that the effective dose of CSE is more accurately related to the amount of bioavailable chemicals per cell. In particular, interactions of CSE components both with cells and other physical factors limit the bioavailabity of CSE (11). According to their report, we suggest that there is a suitable dose of CSE to show its anti-metastatic action in a mouse model of metastasis.

Invasion and migration are indices of the metastatic ability of tumor cells. According to our experimental results, CSE and one of its components, MVK, significantly reduced the invasion by Colon-26 cells, while they did not show any effects on the migration of cells. We suggest that CSE and MVK may reduce the activity of matrix metalloproteinases (MMPs) released from Colon-26 cells and thereby reduce the invasive action of cells without inhibiting their mobility. Rocca et al. reported that cigarette smoke exposure inhibited extracellular MMP2 activity in human lung fibroblasts (HFL1 cell line), and they reported that such an event reflected defective repair of the extracellular matrix (12). We consider that their data support our speculation. Irrespective of this, CSE showed an anti-metastatic action via inhibition of the invasiveness of cells, and MVK is a candidate component of CSE for this action. We highlighted MVK as an active component of CSE, and have already reported that MVK reduced B16-BL6 mouse melanoma cell viability in a concentration-dependent manner in vitro. Mass spectral data showed that the major product formed in cells exposed to CSE was the GSH-MVK adduct via Michael addition. This evidence indicates that MVK found in CSE reacts with GSH in cells to form the GSH-MVK adduct, and thus one possible reason for CSE-induced cytotoxicity is a decrease 
in intracellular GSH level (9). We are planning to investigate the effect of MVK on the experimental hepatic metastasis model in the near future.

In conclusion, CSE significantly reduced metastatic lesions in the liver of experimental hepatic metastasis of Colon-26 cells in syngeneic BALB/c mice. Moreover, CSE significantly inhibited the invasiveness of Colon-26 cells in vitro. Furthermore, MVK may be a candidate active component of CSE since it also inhibited the invasiveness of Colon-26 cells.

\section{Conflicts of Interests}

The Authors declare no conflict of interest associated with this manuscript.

\section{Acknowledgements}

This study was supported by a Special Grant from the Smoking Research Foundation for Biomedical Research of Japan.

\section{References}

1 Saito Y, Takizawa H, Konishi S, Yoshida D and Mizusaki S: Identification of cembratriene-4,6-diol as antitumor-promoting agent from cigarette smoke. Carcinogenesis 6: 1189-1194,1985

2 Roberts DL: Natural tobacco flavor. Recent Adv Tob Sci 14: 4981, 1988.

3 Sees KL: Cigarette smoking, nicotine dependence, and treatment. West J Med 152: 578-584, 1990.

4 Kabat GC: Fifty years' experience of reduced-tar cigarettes: what do we know about their health effects? Inhal Toxicol 15 : 1059-1102, 2003.
5 Breheny D, Zhang H and Massey ED: Application of a two-stage Syrian hamster embryo cell transformation assay to cigarette smoke particulate matter. Mutat Res 572: 45-57, 2005.

6 Toda $\mathrm{N}$ and Toda $\mathrm{H}$ : Nitric oxide-mediate blood flow regulation as affected by smoking and nicotine. Eur J Pharmacol 649: 113,2010

7 Holbrook BD: The effects of nicotine on human fetal development. Birth Defects Res C Embryo Today 108: 181-192, 2016.

8 Takahashi Y, Horiyama S, Kimoto Y, Yoshikawa N, Kunitomo M, Kagota S, Shinozuka K and Nakamura K: Inhibitory effect of cigarette smoke extract on experimental lung metastasis of mouse melanoma by suppressing tumor invasion. Pharmacol Pharmacy 3: 324-329, 2012.

9 Horiyama S, Takahashi Y, Hatai M, Honda C, Suwa K, Ichikawa A, Yoshikawa N, Nakamura K, Kunitomo M, Date S, Masujima $\mathrm{T}$ and Takayama M: Methyl vinyl ketone, a toxic ingredient in cigarette smoke extract, modifies glutathione in mouse melanoma cells. Chem Pharm Bull 62: 772-778, 2014.

10 Higashi T, Mai Y, Noya Y, Horinouchi T, Terada K, Hoshi A, Nepal P, Harada T, Horiguchi M, Hatate C, Kuge Y and Miwa $\mathrm{S}$ : A simple and rapid method for standard preparation of gas phase extract of cigarette smoke. PLoS One 9: e107856, 2014.

11 Bourgeois JS, Jacob J, Garewal A, Ndahayo R and Paxson J: The bioavailability of soluble cigarette smoke extract is reduced through interactions with cells and affects the cellular response to CSE exposure. PLoS One 11: e0163182, 2016.

12 Rocca GL, Anzalone R, Magno F, Farina F, Cappello F and Zummo G: Cigarette smoke exposure inhibits extracellular MMP-2 (gelatinase A) activity in human lung fibroblasts. Respir Res 8: 23, 2007.

Received January 15, 2018

Revised February 12, 2018

Accepted February 13, 2018 